\title{
A Non-Naturalised Methodology for Social Sciences
}

\author{
Omobola Olufunto Badejo \\ http://dx.doi./org/10.4314/ujah.v20i2.9
}

\begin{abstract}
At the rise of the twentieth century, armed with the success of natural sciences, the school of naturalism argued that the appropriate methodology for all disciplines, including social sciences, is that of natural science. The paper argued that social sciences cannot be naturalised and has its own appropriate methodology. The paper examined the arguments for naturalism and non-naturalism of the method of philosophy of social sciences. The paper employed both primary and secondary sources of data. Data collected were subjected to critical analysis and philosophical argumentation. The results showed that the nature of social sciences is such that it cannot be subjected to only scientific methods. The paper concludes that there is a need for a methodology that understands the subject matter of social sciences to address issues in social sciences. The paper addressed some key issues in philosophy of social sciences.
\end{abstract}

Keywords: Methodology, Natural sciences, Naturalism, Social sciences.

\section{Introduction}

Social science, for example history, is the rational study of various aspects of human society and human behaviour with the aim of understanding social phenomena. Social sciences in this paper refers to non-science disciplines such as history and philosophy related to man/woman, his/her environment, social interactions and 
so on. Social sciences are not non science. They are also sciences when understood from the body of knowledge which has its method of arriving at its conclusion. There is an interesting controversy surrounding the appropriate methodology for this rational study. There are basically two schools of thought involved in the debates around the controversy. There is a school of thought that argue that given the success realised within natural science disciplines, for example, physics, the methodology used to arrive at results in the natural sciences should be extended to the social sciences.

This school of thought argue that when the methodology used in the natural sciences is extended to the social sciences, a level of certainty and reliability would be realized in the social sciences. Advocates of this school of thought argue in favour of naturalism of the social sciences. The argument is that the social sciences should adopt the methodology of the natural sciences. This school of thought holds on to the doctrine that all phenomena should be reducible to the laws of physical entities, such as the law of motion. For the purpose of this paper this school of thought will be called the naturalists.

However, there is another school of thought that argues that social science disciplines are not in any way inferior to other disciplines. This school of thought although appreciate the certainty realised in the natural sciences, argue that the subject matter of the natural sciences is different from the subject matter of the social sciences. Hence, it is not possible that the same methodology will suffice for both the natural sciences and the social sciences. This school of thought argue that a different methodology should be added to that of the natural sciences in order to make good progress in the social sciences. For the purpose 
of this paper this school of thought will be called the nonnaturalists.

This paper will add its voice to that of the non-naturalists. The paper will argue that to arrive at rational knowledge, it is not possible for the social sciences to adopt or emulate the methodology of the natural sciences only. The paper will examine some arguments for and against the two schools of thought and also advance arguments to justify non-naturalism of the social sciences.

\section{Naturalism of the Social Sciences}

One of the advocates of the naturalists' school of thought is Carl Hempel. This part of the paper will present some of Carl Hempel's arguments as arguments in favour of naturalism of the social sciences. It is understandable that Hempel belongs to the naturalist's school of thought given his background. Although Hempel did some work in Philosophy, he was a renowned mathematician and physicist. This very well explains his acquaintance and preference for the sciences. Hempel is a philosopher of science and one of the logical empiricists (Ayer 415). Logical empiricism is a movement that emphasizes the role and importance of science and its methodology in the society. It is a movement that has its own controversies, but this paper will not address that.

Hempel in his essay titled "The Function of General Laws in History" argued for a methodological unity between the natural sciences and social sciences. According to Hempel:

... general laws have quite some analogous functions in history and in the natural sciences, that they form an indispensable instrument of historical research, and that they even constitute the basis of various procedures which 
are often considered as characteristic of the social in contradistinction to the natural sciences (Hempel 43).

Hempel argues here that general laws are not only indispensable to the social sciences, but they already play a significant and indispensable role in the social sciences. He believes that for an explanation to be valid, it has to conform to some sort of general laws; "The use of universal empirical hypothesis as explanatory principles distinguishes genuine from pseudo-explanation" (Hempel 45). Hence, to make historical explanations valid, there is a need to properly unify the methodology of the social sciences with that of the natural sciences.

The naturalist school of thought argue that historical explanations actually make recourse to universal hypotheses, which are based on general laws, several times, but hardly acknowledge this. Hempel, for example, listed some words that indicate the use of universal hypothesis in social science explanations; "hence", "therefore", "because", "naturally", "obviously" and "consequently". According to him these words suggest to a listener or reader that inference is being made from a universal law.

Historical explanation, too, aims at showing that the event in question was not a matter of chance, but was to be expected in view of certain antecedent or simultaneous conditions. The expectation referred to is not prophesy or divination, but rational scientific anticipation which rests on the assumption of general laws (Hempel 46).

He further argued that when an explanation is given without stating the general law or universal hypothesis underlying 
the explanation, the explanation is incomplete. In some cases, this omission may not be essential, but in some other cases it is very essential (Hempel 46). Consider this example; the explanation that "a certain political movement has spectacular success because it takes the advantage of racial prejudices" (Hempel 46). This explanation, according to him is incomplete because the universal hypothesis; any political movement that takes advantage of racial prejudices will have spectacular success, is missing from the explanation. Therefore, there is a missing gap between the cause advantage of racial prejudice and the effect - the spectacular success of a certain political movement.

This missing gap is the universal hypothesis that is usually not included in the explanation. Thus, it is this missing gap that is responsible for the ignorance of many in acknowledging that the methodology of the social sciences is not too different from the methodology of the natural sciences. The hypothesis the social scientist uses in his or her explanation is usually not included in that explanation.

According to Hempel, these universal hypotheses are just like universal laws because they are grounded in experience. For example, the universal hypotheses "any political movement that takes advantage of racial prejudices will have spectacular success" is grounded in the knowledge that in the past political movement that took advantage of racial prejudices had spectacular success, hence the historian can draw the inference that a certain political movement recorded spectacular success because they took advantage of racial prejudices. For him, this is the same methodology the natural sciences adopt; the cause and effect relationship.

According to the naturalist school of thought, the claim by some social scientists that the methodology of the social sciences is 
totally different from that of the empirical sciences cannot hold. This is because some social scientists argue that to explain an event in the social sciences, the social scientist employs the method of empathy. By empathy, the historian puts himself or herself in the shoes of the actors in the past event. The historian then imagines the circumstances under which the event took place and comes up with an explanation for why events turned out the way it did.

... he tries to realize as completely as possible the circumstances under which they acted and the motives which influenced their actions; and by this imaginary selfidentification with his heroes he arrives at an understanding and thus at an adequate explanation of the events with which he is concerned (Hempel 49).

The naturalists argue that the above argument cannot subsist as a methodology because it is dispensable and different from the explanation itself. They insist that it is possible for a social scientist to employ this act in arriving at an explanation, but the act is independent of the explanation. Hence, empathy should not exclude a universal hypothesis. Empathy is just one of the devices used to arrive at the explanation, the social scientist would still in his explanation use universal hypotheses to justify why the actor acted in one way and not another (Hempel 49).

Hempel, as a naturalist, also argued that it is possible for a social scientist to arrive at a rational explanation without employing the act. A historian may find it impossible to put himself in the shoes of the actor of an event. In this case, the historian's explanation would be independent of the act of empathy. He argued that since the act is dispensable to 
explanations in the social sciences, it cannot be accepted as the methodology employed by social scientists.

For him, that the social sciences seem to have independent laws, for instance historical laws and sociological laws cannot deny that these laws are derived from generalisations of experience derived from daily activities.

Many of the universal hypotheses underlying historical explanations, for instance, would commonly be classified as psychological, economical, sociological and partly perhaps as historical laws; in addition, historical research has frequently to resort to general laws established in physics, chemistry, and biology (Hempel 52).

Consider this example of such laws: "The use of tree rings in dating events in history rests on the application of certain biological regularities" (Hempel 52). He proceeded to give some reasons that can explain why social scientists fail to properly outline or acknowledge the universal hypothesis underlying their explanations. The first reason is that the universal hypothesis are usually very common and known to many people, hence these universal hypothesis are taken for granted.

First, the universal hypotheses in question frequently relate to individual or social psychology, which somehow is supposed to be familiar with everybody through his everyday experience; thus, they are tactically taken for granted (Hempel 47).

He seems to argue here that social scientists make use of universal hypothesis several times without realising it. The second reason why social scientists usually fail to acknowledge the universal hypothesis underlying their explanation is that it may be necessary to reformulate the hypothesis used such that it will 
conform to the empirical evidence underlying it; but this reformulation is not easy to do.

Second, it would often be very difficult to formulate the underlying assumptions explicitly with sufficient precision and at the same time in such a way that they are in agreement with all the relevant empirical evidence available (Hempel 47).

According to naturalists, though the universal hypotheses the social scientists adopt are like general laws that scientists make recourse to, there is still at least one difference that makes it difficult for universal hypotheses to be formulated like general laws. The difference between these universal hypotheses and the general laws is that general laws are accurately confirmed or can be accurately confirmed, while the confirmation of universal hypotheses cannot be as accurate as that of general laws. This is why it is difficult to formulate universal hypotheses into statements of general laws.

Consider this example; water boils at $100^{\circ} \mathrm{C}$ is a general law used in the natural sciences. An attempt to confirm this law, using the required conditions, will produce an accurate result. However, to confirm the universal hypothesis; "any political movement that takes advantage of racial prejudice will record outstanding success"; there is no guaranty that the result will be accurate.

The illustration used in the preceding paragraph implies that it appears that the universal hypotheses social scientists adopt are mere probabilistic laws (Hempel 47). To make the universal hypotheses used in the social sciences highly probable and closer to accurate general laws, the naturalists introduced the 'explanatory sketch' (Hempel 48). The naturalists argue that the 
reason why it seems the hypotheses used in the social sciences are so probabilistic is because the explanation given by the social sciences are sketches that needs some filling out.

Naturalists insist that even in the natural sciences, probabilistic laws are used; however, the explanations given in such cases go beyond mere sketches. Hempel used an example to illustrate this:

Thus, e.g., if Tommy comes down with the measles two weeks after his brother, and if he has not been in the company of other persons having the measles, we accept the explanation that he caught the disease from his brother. Now, there is a general law underlying this explanation; but it can hardly be said to be a general law to the effect that any person who has not had the measles before will get it without fail if he stays in the company of somebody else who has the measles; that contagion will occur can be asserted only with high probability (Hempel 48).

According to naturalists, the argument that explanations in the social sciences use probabilistic laws is not evidence that the methodology of the social sciences differ radically from that of the natural sciences. What is needed in the social sciences, to avoid 'pseudo-explanations', is in assessing an explanation to try as much as possible to identify and formulate the hypotheses underlying the assertions made in the explanation.

For naturalists, the role of a social scientist is not purely descriptive, after explaining an event; the social scientist offers explanations for why events took that course and not some other. Hempel considers the latter part of the explanation as the most important part of the explanation and this part is deeply rooted in experience and grounded in various universal hypotheses. Hence, 
the separation of the methodology of the social sciences from that of the natural sciences is wrong and uncalled for, according to him. This can be inferred from his concluding remarks:

The necessity, in historical inquiry, to make extensive use of universal hypotheses of which at least the overwhelming majority come from fields of research traditionally distinguished from history is just one of the aspects of what may be called the methodological unity of empirical science (Hempel 52).

The argument of the naturalists is that contrary to the claim of social scientists, there is no unique methodology for social sciences different from that of science. Hence, there can be a methodological unity between science and social sciences. To achieve this, the methodology of the social sciences should be naturalised. The argument of this school of thought hinges largely on the assumption that general or universal laws are indispensable to any discipline, as it distinguishes genuine from pseudo theories, explanations and hypothesis. However, the non-naturalist school of thought disagree with this position.

\section{Non-naturalism of the Social Sciences}

The non-naturalists disagree with the naturalist school of thought that there can be a methodological unity between science and social sciences. This is because the nature of the natural sciences differ largely from the nature of the social sciences; hence they cannot use the same methodology to produce results.

One of the justifications for a different methodology for the social sciences from the natural sciences stems from the distinction between the nature of the social sciences and the nature of the sciences. According to Friedrich Hayek for example, the natural 
sciences study the aspects of nature that are easily and immediately presented to the senses. The senses can easily recognise the recurrent patterns in nature, and then questions are asked on how and why these patterns are recurrent in nature. There are other recurrent patterns in nature that the senses cannot comprehend; these patterns are recognised only when reasoning comes into play. Example of such patterns is mathematics:

Marvellous, however, as the intuitive capacity of our senses for pattern recognition is, it is still limited. Only certain kinds of regular arrangements (not necessarily the simplest) obtrude themselves on our senses. Many of the patterns of nature we can discover only after they have been constructed by our mind (Hayek 55).

According to Hayek, the natural sciences study strictly the physical phenomena of nature, while the social sciences study the non-physical phenomena of nature. He argued that the difference in the degree of complexity between both fields of study is inherent in the distinction in the aspects of nature that they seek to understand (Hayek 57-58). Thus, "Non-physical phenomena are more complex because we call physical what can be described by relatively simple formulas" (Hayek 57). The implication of this is that the same methodology used to understand simple phenomena cannot be used to understand complex phenomena.

Simple phenomena consist of an intuitive ability to recognize patterns and events in nature. This recognition of certain patterns and events usually leads to a general law or theory. Simple phenomena are subject to the cause and effect chain. However, in the social sciences what obtains is more of recognition of individual patterns and events. This is not usually subject to the cause and effect chain. When the cause and effect chain is not 
involved in the study of an event, it is more difficult to understand the event. Naturalists such as Francesco Guala argue that social phenomena can be constructed to align with general laws (Guala 45).

However, a question arises from Hayek's distinction between the natural sciences and the social sciences based on the degree of complexity. The question is "how can we measure the degree of complexity in regards to generalisations or theories? The degree of complexity can be measured by the number of data that is sufficient to arrive at conclusions in the natural sciences and in the social sciences.

Yet when we consider the question from the angle of the minimum number of distinct variables a formula or model must possess in order to reproduce the characteristic patterns of structures of different fields (or to exhibit the general laws which these structures obey), the increasing complexity as we proceed from the inanimate to the ("more highly organized") animate and social phenomena becomes fairly obvious (Hayek 56-57).

According to Hayek, when the general laws governing the natural sciences are examined it will be discovered that from few variables a conclusion can be arrived at and that other variables will be subject to that general law. This is unlike what obtains in the social sciences. When we deal with inanimate phenomena, it is easier to arrive at conclusions. Consider the law of gravity that states that "everything that goes up must come down". It is not necessary to consider so many variables to arrive at a consistent pattern as regards the law of gravity. However, in the social sciences more variables must be considered before a phenomenon can be understood or described. 
Hayek's first justification of the degree of complexity in the natural sciences and in the social sciences is in the number of variables sufficient to make assertions. The second justification is that in the natural sciences the more the variables introduced in predicting a pattern, the more consistent the prediction of that pattern will be. However, in the social sciences the more the variables introduced in predicting a pattern, the more inconsistent and accidental the pattern will appear to be.

Hence, the argument that natural sciences are successful because it has been able to discover regularities is false. The ability of the natural sciences to discover regularities easily is because it deals with simple phenomena and not because it is superior to other disciplines. The third justification for the distinction in the degree of complexity, is that there is no need to gather information about individual data to generalise in the natural sciences. Hayek argued that in the natural sciences general properties of the variables are sufficient to make a generalisation. A general law is still applicable to individual variables as long as the variable possesses the general properties defined by the scope of that general law (Hayek 58). In the social sciences however, individual events, objects or variables depend on so many circumstances, all important, that it is difficult to achieve the type of certainty achieved in the natural sciences.

The more we transcend from the study of inanimate phenomena into animate and social phenomena; the less likely that the degree of certainty achieved with animate phenomena will be achieved. The natural sciences merely recognise and describe certain patterns or order, but cannot explain individual events. Data or variables used in the natural sciences are physical objects; these physical objects are not dependent on personal beliefs, values and perceptions. However in the social sciences, the data or variables 
used are non-physical social objects. These social objects are formed by personal beliefs, values and perceptions. Hence, it is not possible to understand physical objects the same way physical objects would be understood. Physical objects are subject to simple formulae, while non-physical objects are subject to more complex formulae. Hayek argued that based on these fundamental differences, the social sciences cannot be subjected to the methodology of the natural sciences.

For non-naturalists, it is impossible to generate results in the social sciences if it is restricted to the study of certain patterns or events. The study of individual patterns and events cannot be separated from the social sciences. Attempts to seek prediction of simple regularities in the social sciences are futile. The social sciences are confronted with individual data that are subject to many variations and circumstances. To attain exact predictions, it is necessary to know all the variations and circumstances each individual data is subject to. However, it is not possible to ascertain all the variations and circumstances that the data is subject to. This is because of the subjectivity of each data. The natural sciences can attain exact prediction because they are confronted with patterns consisting of few and the same variations under the same circumstances. Hence, it is easy to ascertain these variations and circumstances and generalise over them.

The closest the social sciences can achieve in generating laws is in economics. As much as economics has succeeded in generating laws and theories, economics cannot claim to have all the information on the data generalised over.

... economic theory is confined to describing kinds of patterns which will appear if certain general conditions are satisfied, but can rarely if ever derive from this knowledge any predictions of specific phenomena (Hayek 62). 
It is a delusion to assume that every phenomenon is subject to simple regularities. To make progress in the social sciences, any attempt to obtain prediction of simple regularities must be stopped. The social sciences are not supposed to identify cause and effect relations, but ought to explain social phenomena. Hence, it is wrong to keep trying to produce 'laws' in the sense that the natural sciences do (Hayek 66-67).

Another advocate of the non-naturalists; Michael Scriven argued along the same line as Hayek, though in a more subtle form (Scriven 71-76). Both Hayek and Scriven argued that the natural sciences are not more important than or superior to the social sciences but that the social sciences are faced with complex challenges, while that of the sciences are simple challenges.

The difference between the scientific study of behavior and that of physical phenomena is thus partly due to the relatively greater complexity of the simplest phenomena we are concerned to account for in a behaviorial theory (Scriven 72).

According to Scriven, given the nature of the social sciences; the methodology of the natural sciences cannot suffice for the social sciences. The subject of the social sciences is largely human beings and understanding human beings is a more complex task than understanding inanimate objects; which is largely the subject of the natural sciences (Scriven 75). Non-naturalists acknowledge that there is no science; either natural sciences or social sciences that do not make recourse to observation and prediction at some point. However, recourse to observation and prediction in the natural sciences is simple and regular. This is because the subject of the natural sciences is largely inanimate objects. Inanimate objects are not subject to variations and 
individual straits like animate objects. In the social sciences however recourse to observation and prediction is less regular. The method of the social sciences is largely explanation.

Thus, there is no reason to expect that one day the methodology of the natural sciences can be made to fit into the social sciences. The nature of the social sciences is such that any aspect of it that fully obeys simple regularities is no longer an aspect of the social sciences but becomes an aspect of the natural sciences. In other words, even with the huge level of certainty and reliability realised in the sciences; there are problems that the natural sciences cannot solve, and will not attempt to solve. When this happens the natural sciences advance by abandoning such problems. For example, physical sciences will not embark on the project of comparing religions.

Given the arguments of Scriven and Hayek for nonnaturalism of the social science, it is obvious that what the social sciences address is more complex than what the natural sciences address. Hence, there is no justification for the attempts to impose the methodology used in the natural sciences on the social sciences. The natural sciences depend solely on empirical approach, while the social sciences employ the empirical approach, powers of reason and logical argument. The point of contention is that the nature of the social science cannot allow it to fit strictly into the empirical approach.

The empirical method is experiment; that is independent of the observer, dependent on results and extraneous control over variables. Findings are generated in the social sciences with the careful objective of establishing cause and effect relationship. Personal feelings and experiences are useless in scientific natural findings. Future behaviours of variables in a set or class can be predicted from the findings of the research carried out on a fraction 
of the variables in that set. The theory produced from this research easily becomes a general law. A 'general' law in the sense that it can be tested, it can be replicated under the same conditions and is not ambiguous. This empirical approach is what gives the reliability and the much spoken of success in the natural sciences.

Consider this illustration "water boils at $100^{\circ} \mathrm{C}$ " is a general law. The cause and effect relationship in this law is that when water is subjected to heat of up to $100^{\circ} \mathrm{C}$ it will boil. The cause is the heat the water is subjected to, while the effect is the boiling of the water. This general law can be repeated under the same conditions and the result will be the same. This is because neither water nor heat has the ability to change themselves. This is the major factor that makes reliability possible in the natural sciences. The reliability being celebrated in the natural sciences is not a function of a superior methodology, but a function of the simple nature of the data being studied.

Hence, the naturalists made a fundamental error by embarking on a journey of naturalising the methodology of social sciences, rather than a journey of understanding the differences in the nature of science and social sciences. My first challenge with the naturalist school of thought is that they failed to address in their arguments the nature of the social sciences. Before the question of whether the methodology of the natural sciences is appropriate for the social sciences, it is necessary to first understand the nature of the social sciences and the differences between it and that of science.

The first quest should be "what are the questions the social sciences address?" Just as the definition of the social sciences suggests; the social sciences, for the purpose of understanding and explanation, study various aspects of human society, human behaviour and social phenomenon. From this description of the 
nature of the social sciences, it is obvious that while the natural sciences study physical entities, the social sciences study social phenomena. The subject matter of the former is entirely physical and open to empirical observation, while the subject matter of the latter is not entirely physical and some aspects of it are not open to empirical observation.

Consider the illustration given by Hempel; “... or that a certain political movement has spectacular success "because" it takes advantage of widespread racial prejudices" (Hempel 46). Hempel used this example to argue that the social science is not devoid of the cause and effect relationship and I doubt if any serious social scientist would argue otherwise. The issue here is not that it is not expected that any political movement that takes advantage of widespread racial prejudice would have spectacular success.

The issue is that it cannot be surprising or shocking if a political movement satisfies that condition and still do not have spectacular success. There may be some questions arising from it, but it cannot be a major point of concern. However, if water fails to boil at $100^{\circ} \mathrm{C}$, then it would be a major point of concern. It would be a major event in every part of the world and the head line on every tabloid. This is because the data of the natural sciences is not expected to be subject to variations like the data of the social sciences. This is one of the reasons why a social scientist would do more than just expect the future to be like the past.

According to Wilhelm Dilthey in his essay titled "The Understanding of Other Persons and Their Life Expressions", Dilthey argued that human beings; their actions and behaviour are the subject matter of the social sciences. This subject matter has two aspects; the mental and the physical aspects. The physical aspect is open to the senses, hence can be understood and 
explained using the empirical approach. However, the mental aspect is not open to the senses and can be understood using the reasoning approach (Dilthey 214-215).

For him, the mental phenomenon; which is the main subject matter of the social sciences is more important than the physical aspect because the mental aspect determines the physical aspect. This is without prejudice to Dilthey's acknowledgement that the social science is not devoid of the physical aspect. This shows that the social science from time to time appeal to the empirical approach, but is not restricted to the empirical approach. His argument is evidence that the non-naturalists are not denying that the social sciences need to employ the empirical approach at one time or the other.

Robin Collingwood argued in the same line as Dilthey. Collingwood argued that the natural sciences study the outside of events; this is synonymous to the physical phenomena. While the social sciences study both the outside and the inside of events; the inside is synonymous to the mental phenomena. The natural sciences have no outside (Collinwood, 251). This is another evidence that the non-naturalists acknowledge that the methodology of the natural sciences is useful in the social sciences.

The argument I am trying to draw out in favour of nonnaturalism of the methodology of the social sciences is not that the methodology of the natural sciences is useless in the social sciences, but naturalism of methodology cannot lead to good progress in the social sciences. This is mainly because the nature of the data of the social sciences differs from that of the natural sciences to a large extent. Animals by nature are capable of pretence, especially human beings, but inanimate objects like water is not capable of pretence. If water had the same nature as human behaviour, I am sure that the general law water boils at $100^{\circ} \mathrm{C}$ 
would be a myth today. Of course, animals have the physical aspects that can be understood and explained objectively by the natural sciences. It is the non-physical aspect of man, which is behaviour, which constitutes social phenomena.

A second challenge with the position of the naturalist is that it seems their centres more on an assumption that the nonnaturalists out rightly reject the methodology of the natural sciences. This assumption is erroneous; the argument of the nonnaturalists is that the methodology of the natural sciences is not adequate to produce results in the social sciences. Hence, the social sciences employ the method of reasoning and logic. There is no justification for the assumption that all phenomena must be completely subject to the empirical approach. Daniel Little calls this assumption a basic error and a fallacy of naturalism (Little 13). An attempt to naturalise non science disciplines is more or less an appeal to verificationism that tried to reduce meaningful statements to those that are only verifiable empirically. This is erroneous and largely discredited. Furthermore,

The third challenge with the naturalists' position is with the explanatory sketch. Hempel's explanatory sketch seems laughable. Even in the natural sciences, explanations are not given by adding every detail used to generate results; yet the explanations are not considered incomplete. That an explanation in the social sciences seems incomplete when subjected strictly to experience shows that experience alone cannot give proper and meaningful explanations in the social sciences. The social science will continue to take a back seat except it adopts its own methodology.

Furthermore, to assume a complete theory, explanation or hypothesis is one that relies on a universal law is erroneous. Science itself, though depends on universal laws, is not immune to revision. Science is open to revision and some of its hypothesis has 
undergone revision. Science is aware that reliance on universal or general laws are not immune to changes. For example, there was a time when it was a scientific fact that the tongue has specific receptor areas for specific flavours. However, it is now a scientific fact that there is no specific receptor area for any flavour in the tongue. Every receptor in the tongue can sense all flavours.

Furthermore, the type of laws used in the natural sciences is causal laws. These causal laws are universal in nature; they are independent and not controlled by time or location. As Hempel argued, it is true that there are some social laws, but they cannot be elevated to the status of general laws. This is because they lack the independence that characterises general laws. An example of this is the law of demand in economics. Though it is a widely accepted law; it is dependent on many variables and lack the reliability that governs general laws. The law of demand is also weak in comparison with the law of gravity; the law of demand cannot predict the magnitude of the expected change in demand and supply. The human behaviour and social world is too complex to expect regularities as in the natural sciences.

A third challenge with the position of naturalists on the methodology of social science is the argument that the use of words such as 'hence', 'naturally' and 'therefore' is an evidence that social science already depends heavily on universal laws. The assumption that these words points to general laws or universal laws is erroneous. Naturalists who argue along this line lack the knowledge of basic logic. Basic logic shows that these words are mere claim locators and not evidence of any universal laws. A claim locator merely shows the inference made from a set of statements to a claim. The set of statements can be universal, general or merely explanatory and not necessarily an indicator of the presence of a general law. 
A fourth challenge to the argument that the methodology of the social sciences should be naturalised lies in the complex nature of the subject matter of social science disciplines. Of course, the reliability in the natural sciences is impressive and reduces to the barest minimum disagreements arising from interpretations of events. However, this reliability is possible, not because the natural science is superior to the social science. The reliability is possible because the subject matter of the natural sciences is simpler than the subject matter of the social sciences. This distinction occurs because the data used in the natural sciences are largely objective, while the data of the social sciences are largely subjective.

The methodology of the natural sciences simply cannot suffice for the social sciences. As Hayek argued, "This is the price we have to pay for an advance into the field of complex phenomena" (Hayek 58). There is no justification for the assumption that because of the level of success experienced in the natural sciences, then the social sciences too should be done like the natural sciences. I agree with Hayek's argument that the social science cannot make progress if it keeps living in the shadow of the natural sciences.

What we must get rid of is the naïve superstition that the world must be so organized that it is possible by direct observation to discover simple regularities between all phenomena and that this is a necessary presupposition for the application of the scientific method... if we want to get ahead in these fields our aim will have to be somewhat different from what they are in the fields of simple phenomena (Hayek 66).

The question arising from the position of the nonnaturalists is "What methodology then is appropriate for the social 
sciences?" It cannot be surprising if the search for the methodology appropriate for the social sciences raises further disagreements. Natural scientists agree on the methodology appropriate for the natural sciences. However, social scientists may not easily get a consensus. This problem is still an off shoot of the nature of the subject matter of the social sciences. Because the data of the social sciences is largely subjective and subject to variations to a large extent; how to study and understand them will also be subjective and open to variations. Hence, it will still generate questions and controversies. This question is another broad and interesting topic on its own, which the scope of this essay will not cover. A lot has been done and is still being done on addressing the question and controversy of the methodology appropriate for the social sciences.

Two instances of these methodologies are hermeneutics and descriptivism. Descriptivism argues that the methodology appropriate for the social sciences is to re-enact and re-express the beliefs, values and understanding of the society through the point of view of the inhabitant of these beliefs, values and understanding of social phenomena (See McIntyre 453-464, Dilthey, Grodin).These are not necessarily all that is to the methodology of the social scientists. Descriptivism is just an aspect of the methodology of the social sciences. There is Hermeneutics which is also a methodology in the social sciences. Hermeneutics goes beyond just re-enacting through the eyes of the inhabitants. Hermeneutics extends to comparing and contrasting these reenacted views, beliefs and values with that of the presenter or with contemporary views, beliefs and value (See Collingwood, Bishop). Hermeneutics prescribes describing and interpretation as important aspects of the methodology of the social sciences (Olesen7-157). 


\section{Conclusion}

I will conclude by reaffirming the positions of this paper; first, the methodology of the natural sciences is not sufficient for the social sciences given the striking difference between their subject matters. Two, the social sciences is not inferior to the natural sciences just because it cannot produce the level of certainty and reliability achieved in the natural sciences. Even science is openended and not immune to revision. Three, there is no justification for the argument that all phenomenon should adopt the methodology of the natural sciences. Four, to make good progress, the social sciences must adopt its own methodology different from that of the natural sciences. Five, the social scientists are not rejecting the empirical approach out rightly. The social scientists adopt the empirical approach where it is possible and necessary. However, the methodology of the social sciences allows for further interrogation even in the absence of empirical evidence.

Therefore, an attempt to force the social sciences to fit into the methodology of the social sciences is to force a square peg into a round hole. If the subject matter of the natural sciences and the social sciences differ sharply, then it is not expected that the methodology of one should be necessarily appropriate for the other. The role of the natural sciences is to describe and explain the physical aspect of the world. This role is neutral when it comes to making judgements about values. However, the social sciences seek to understand and explain the non-physical aspect of the world; sometimes in relation with the physical. This role is not neutral when it comes to value judgements; value judgement is a huge task in the social sciences. This role is without prejudice to the empirical approach where necessary.

Even science disciplines have instances where they need to depend on value judgement and not general laws. For example, in 
medical sciences, health workers are not only concerned about general laws in dealing with the health of patients, but also value laden judgement to understand the behaviour of their patients and how their behaviours may affect their health plan. The complexity of the data of the social scientists makes controlled experiments difficult or out rightly impossible in some cases. Hence, social sciences reserves the right to employ controlled experiments, that is the methodology of sciences, where possible and also employ non-controlled experiments when there is a need to deal with more complex phenomena.

Omobola Olufunto Badejo

Department of Philosophy

Obafemi Awolowo University, Ile-Ife

funtoarawomo@gmail.com 


\section{References}

Ayer, A.J. Language, Truth and Logic. London: Victor Gollanancz, 1946.

Bishop, Robert. The Philosophy of the Social Sciences. New York: Continuum, 2007.

Collingwood, Robin. "History as Re-enactment of Past Experience." Theories of History. Ed. Patrick Gardiner. New York: Free Press, 1959.

Dilthey, Wilhelm. "The Nature of Historical Knowledge." Theories of History. Ed. Patrick Gardiner. New York: Free Press, 1959.

Grodin Joseph. Introduction to Philosophical Hermeneutics. Trans by Weinsheimer J. New Haven: Yale Uni. Press, 1997.

Guala, Francesco. "Philosophy of the Social Sciences: Naturalism \& Anti-Naturalism in the Philosophy of Social Sciences." The Oxford Handbook of Philosophy of Science. Ed. Paul Humprey. Madison Avenue: OUP, 2016, pp.43-64.

Hayek, Friedrich. "The Theory of Complex Phenomena" Readings in the Philosophy of Social Science. Eds. Michael Martin and Lee C. McIntyre. Cambridge: MIT Press, 1994, pp.55 70.

Hempel, Carl. "The Function of General Laws in History." Readings in the Philosophy of Social Science. Eds. Michael Martin and Lee C. McIntyre. Cambridge: MIT Press, 1994, pp.43-54.

Little, Daniel. New Directions in the Philosophy of Social Sciences. Stannary Street: Rowman \& Littlefield: 2016.

McIntyre Lee. "Redescription and descriptivism in the Social Sciences." Behaviour and Philosophy, Vol. 32, No.2, 2004: 453-464. 
Olesen, Henning. "Cultural Analysis and In-Depth Hermeneutics." Historical Social Research Focus, Vol.38 No.2, 2013: 7157.

Scriven, Michael "A Possible Distinction between Traditional Scientific Disciplines and the Study of Human Behavior" Readings in the Philosophy of Social Science. Eds. Michael Martin and Lee C. McIntyre Cambridge: MIT Press, pp.718.

Winch, Peter. The Idea of a Social Science and its Relation to Philosophy. NJ: Humanities Press Inc., 1958. 